second infusion, W16 and the visit on which a new rituximab cycle was prescribed. The dotted ines represent a DAS28CRP-score of 2.6 (remission cut-off) and 3.2 (low disease activity cutoff). C: cycle; W: week; VAS: visual analogue scale.

Disclosure of Interests: None declared

DOI: 10.1136/annrheumdis-2021-eular.1783

\section{POS0617 ANTI INFLIXIMAB ANTIBODIES DETECTED BY A DRUG TOLERANT ASSAY ARE FREQUENT BUT, IN MANY CASES, WITHOUT RELEVANT CLINICAL SIGNIFICANCE}

A. Martínez-Feito ${ }^{1,2}$, B. Hernández-Breijo ${ }^{2}$, M. Novella-Navarro ${ }^{2,3}$, V. NavarroCompán ${ }^{2,3}$, C. Diego ${ }^{1}$, I. Monjo ${ }^{2}$, L. Nuño ${ }^{2}$, A. Villalva ${ }^{3}$, D. Peiteado ${ }^{3}$, D. Pascual-Salcedo ${ }^{2}$, P. Nozal ${ }^{1}$, A. Balsa ${ }^{2,3}$, C. Plasencia ${ }^{2,3} .{ }^{1}$ La Paz University Hospital, Immunology, MADRID, Spain; ${ }^{2}$ IdiPaz. La Paz University Hospital, Immuno-Rheumatology Research Group, MADRID, Spain; ${ }^{3}$ La Paz University Hospital, Rheumatology, MADRID, Spain

Background: Infliximab (Ifx) has proven effective in treating rheumatoid arthritis (RA) and spondyloarthropathies (SpA), although around $40 \%$ of cases fails, mainly due to immunogenicity. Formation of immunocomplexes between antibodies to Ifx (ATI) and Ifx can increase drug clearance, leading to treatment failure. Standard ELISA assays which are drug -sensitive are frequently used, being able to detect only free ATI. Interest in drug-tolerant assays to measure total ATI (free and complexed) is increasing.

Objectives: To compare the development of ATI using both drug-tolerant and drug-sensitive assays at early stages of Ifx therapy. To analyse the relationship of ATI detected by both assays with the drop-out of treatment.

Methods: This is a prospective observational study including 45 patients with RA and 61 with axial-SpA treated with standard doses of Ifx $(3 \mathrm{mg} / \mathrm{kg}$ and $5 \mathrm{mg} /$ $\mathrm{kg}$, respectively) enrolled at Biological Therapy Unit of Hospital La Paz. Serum samples were obtained at 2, 6, 12 and 22 weeks (W) after Ifx initiation. The data about discontinuation for inefficacy was obtained from the database. ATI presence was evaluated by a drug-sensitive in-house two-site (bridging) ELISA (bELISA) and a drug-tolerant commercial ELISA assay (Immundiagnostik®,IDK) All comparisons were performed throughout non-parametrical test. In SpA group, due to the low number of ATI+ patients at W12 by bELISA the statistical analysis to compare both assays were not performed

Results: ATI detection by both assays at early stages ( $\leq 22 \mathrm{~W})$ of treatment is shown in Table 1a. ATI were always detected earlier by IDK than bELISA and also in RA than in SpA patients probably reflecting the effect of lower Ifx doses. Three out of $106(3 \%)$ vs $0(0 \%)$ patients had ATI at W 2 and $62(58 \%)$ vs $20(18 \%)$ patients at W22, by IDK and bELISA, respectively.

Table 1.

\begin{tabular}{|c|c|c|c|c|c|c|c|c|}
\hline & \multicolumn{2}{|c|}{ W2 } & \multicolumn{2}{|c|}{ W6 } & \multicolumn{2}{|c|}{ W12 } & \multicolumn{2}{|c|}{ W22 } \\
\hline & AR & $\mathrm{SpA}$ & AR & $\mathrm{SpA}$ & AR & $\mathrm{SpA}$ & AR & $\mathrm{SpA}$ \\
\hline \multicolumn{9}{|c|}{ a) ATI+ patients ( $n, \%)$ at early stages } \\
\hline bELISA & 0 & 0 & $3(7 \%)$ & 0 & $10(22 \%)$ & $1(2 \%)$ & $13(29 \%)$ & $7(12 \%)$ \\
\hline IDK & $1(2 \%)$ & $2(3 \%)$ & $7(16 \%)$ & $2(3 \%)$ & $16(36 \%)$ & $16(26 \%)$ & $28(62 \%)$ & $34(56 \%)$ \\
\hline \multicolumn{9}{|c|}{ b) Patients who discontinued $(n, \%)$ Ifx therapy considering ATI status at early stages } \\
\hline bELISA+ & & & & & $9(90 \%)^{*}$ & $1(100 \%)^{*}$ & $12(92 \%)$ & $4(57 \%)$ \\
\hline bELISA- & & & & & $23(66 \%)$ & $22(37 \%)$ & $20(63 \%)$ & $19(35 \%)$ \\
\hline IDK+ & & & & & $15(94 \%)^{\star}$ & $7(44 \%)$ & $24(86 \%)$ & $13(38 \%)$ \\
\hline IDK- & & & & & $17(59 \%)$ & $11(24 \%)$ & $13(77 \%)$ & $10(27 \%)$ \\
\hline
\end{tabular}

${ }^{*} \mathrm{p}<0.05$ comparing between $\mathrm{ATI}+\mathrm{vs}$ ATI- in each assay.

Once ATIs appeared, regardless both methods, they persisted throughout the follow-up, indicating that immunogenicity was not transient.

At W22, only $13 / 28(46 \%)$ and $7 / 34(21 \%)$ patients with ATI detected by IDK were also positive by bELISA in RA and SpA, respectively.

ATI levels by IDK were higher in ATI+ by bELISA than in ATI- patients at early stages: ATI levels by IDK at W12: $91[74-348] \mathrm{ng} / \mathrm{ml} \mathrm{ATI+}$ vs 21.7[15-59.5] ng/ $\mathrm{ml} \mathrm{ATI-}(\mathrm{p}<0.01)$ and at W22: 132 [89-372] ng/ml ATI+ vs 23[13-66] ng/ml ATI$(p<0.001)$. However, only in $4 \%(2 / 45)$ patients with RA and in $13 \%(8 / 61)$ patients with SpA the detection by IDK was earlier than by bELISA at W12. Free IFX in serum was not detected in bELISA ATI+ patients. In IDK ATI+ patients low circulating Ifx levels were present as compare to ATI- since W6 to the end of follow-up $(p<0.01)$

More ATI+ patients dropped out Ifx at $\mathrm{W} 12$ and $\mathrm{W} 22$ regardless de assay (Table 1.b), being statistically significant for both assays in patients with RA and only for bELISA in patients with SpA.

Conclusion: ATI measured by a drug-tolerant assay are always detected earlier than ATI detected by bELISA, indicating that immunogenicity, at least with
Ifx, is usually an early event. High levels of ATI by IDK are associated with an earlier detection by bELISA in case of RA patients. ATI detected only by drug tolerant assays are associated with low levels of circulating Ifx but not with a complete drug neutralization and may do not have clinical relevance compared to ATI detected by bELISA. Many patients have low levels of ATI which can only be detected by drug tolerant assays after long-term of follow-up.

The reasons why ATI levels rise rapidly in some patients while in others remain low are currently unknown but may be relevant if the clinical effect of immunogenicity is to be minimized.

Acknowledgements: We are grateful to all the rheumatologists and nurses of the Daycare Department for Biologics and to the laboratory technicians of the Immunological Unit

Disclosure of Interests: ANA MARTÍNEZ-FEITO: None declared, Borja Hernández-Breijo: None declared, Marta Novella-Navarro: None declared Victoria Navarro-Compán Grant/research support from: AbbVie, Janssen, Lilly, Novartis, Pfizer, and UCB, Cristina Diego: None declared, Irene Monjo: None declared, Laura Nuño: None declared, Alejandro Villalva: None declared, Diana Peiteado: None declared, DORA PASCUAL-SALCEDO: None declared, Pila Nozal: None declared, Alejandro Balsa Grant/research support from: Abbvie, Pfizer, Novartis, Roche.Amgen, Sandoz, Lilly, UCB. Personal fees and nonfinancial support from BMS. Grants, personal fees and non- financial suppor from Nordic., Chamaida Plasencia Grant/research support from: AbbVie, Lilly, Novartis, Pfizer,Sanofi, Biogen and UCB.

DOI: 10.1136/annrheumdis-2021-eular.1791

\section{POS0618 PERSISTENCE OF REMISSION AFTER TAPERING OF GOLIMUMAB IN INFLAMMATORY JOINT DISEASE (IJD)}

A. Damiani ${ }^{1}$, F. Bartoli ${ }^{1}$, V. Gori ${ }^{1}$, S. Bellando-Randone ${ }^{1}$, G. Fiori ${ }^{1}$, M. MatucciCerinic ${ }^{1}$, S. Guiducci' . 'University of Florence, Rheumatology Unit, Firenze, Italy

Background: In refractory IJD, remission may be obtained with antiTNFa drugs and other biological disease modifying anti-rheumatic drugs (bDMARDs). The last EULAR recommendations suggest tapering of bDMARD when remission persists ${ }^{1}$. However, best timing and modality of tapering are uncertain and specific knowledge on patients' characteristics associated to a better outcome is still lacking.

Objectives: To evaluate the persistency of remission after increasing the interval between injections of Golimumab in a group of patients affected by rheumatoid arthritis (RA), psoriatic arthritis (PsA), ankylosing spondylitis (AS) and juvenile idiopathic arthritis (JIA) and to identify any variables associated to disease flare after tapering.

Methods: Between 2011 and July 2020, 80 patients affected by RA, PsA, AS and JIA treated with Golimumab were enrolled. Their demographic and clinical data, including inflammation (ESR and CRP) and clinimetric indices (DAS28 or BASDAI), were collected at baseline and during the follow up visit (T1). In 22/80 patients that reached clinical remission at $\mathrm{T} 1$, the time between Golimumab injections has been prolonged (mean time between injection: 43.7 days); ESR and cRP, DAS28/BASDAI, and time since the start of the tapering (weeks) were evaluated in the next control visit (T2)

Results: 80 patients were enrolled (32 male, mean age 50.6 years +/- 13.91), 34 AS, 33 PsA, 9 RA and 4 JIA. At baseline they have an active disease with a DAS 28 of $4.74+/-0.85$ and a BASDAl of $5.23+/-1.31$. At T1, 60/80 patients were in remission (75\%), with a mean DAS 28 of $1.84+/-0.6$ and an average BASDAI of $1.32+/-0.6$, and 22/60 patients started drug tapering. At T2, 20/22 patients (91\%) were in remission, (DAS $281.9+/-0.49$, BASDAI of $0.8+/-0,67$ ) A significantly higher BASDAl was observed at $\mathrm{T} 1$, even though in the range of absence of disease activity $(2.2,+/-0.28$ vs $0.58,+/-0.47 ; p<0.001)$ in patients who, after extending the therapeutic interval ( $\mathrm{T} 1)$ were no longer in remission at T2. Patients with a flare of disease activity $(2 / 22)$ were taken back to the 28 days window of Golimumab with a prompt recovery of disease remission. Out of the 38 patients maintained at the standard dose, 4 experienced a disease flare with necessity to switch or swope bDMARD, with a retention rate in this group of $90 \%$. Difference of retention rate between patients on standard $v s$ reduced dose was not statistically significative.

Conclusion: Tapering of Golimumab was successful in $91 \%$ of the cases without flare. Moreover, the prolongation of the increase of the treatment window provided the same result as that obtained in patients that continued in the standard time window. This evidence suggests that the extension of the gap between Golimumab administrations may be feasible and safely applied in practice.

\section{REFERENCES:}

[1] Smolen JS, Landewé RBM, Bijlsma JWJ, et al EULAR recommendations for the management of rheumatoid arthritis with synthetic and biological disease-modifying antirheumatic drugs: 2019 update Annals of the Rheumatic Diseases 2020;79:685-699.

Disclosure of Interests: None declared

DOI: 10.1136/annrheumdis-2021-eular.1959 